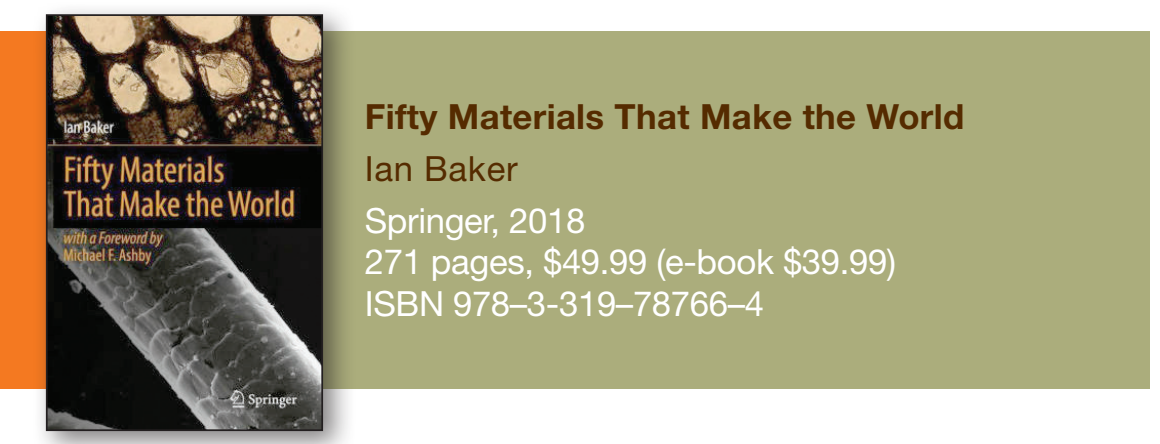

This book could be considered a a concise, semi-technical review of 50 materials of significance to mankind. A combination of metals, plastics, and various naturally occurring materials such as stone and wood are included, and the decision on what to include must have been a difficult one for the author. Surprisingly, materials such as water, air, and soil are left out. The pre-set limit of 50 materials and perhaps the historical context spanning many centuries are the two factors that likely precluded the inclusion of many more recently developed modern materials such as high-temperature superconductors, high-entropy alloys, semiconductors, nanomaterials, and various energy-storage materials, which already greatly influence our lives.

The book contains numerous references for those wanting to dig deeper, including many Internet links as footnotes. The coverage focuses on applications and the practical impact of various materials on our lives, including tidbits of descriptive information and interesting historical context, uncovering little-known background information about the discovery and development of various materials. Also included are basic economics information (production scale by country) and geography data on the occurrence of specific material resources. The reader will learn facts that are often less known yet significant (e.g., that most of the gold is found in seawater or that platinum is about 10 times more common in the earth's crust than gold).

The technical value of the book would be enhanced if, in the case of elements, the description included more details on the atomic structure and known isotopes. On the other hand, the text can be useful in designing thematic travel itineraries, such as a visit to the subterranean London Silver Vaults, to learn about and purchase the largest variety of silver goods not found anywhere else.

The book briefly touches on the subject of "rapid prototyping" (additive manufacturing, AM) in connection with polymers. As materials play a key role in this emerging technology, more detailed coverage of this topic (especially that of metal AM) would further enhance the next edition of this book.

Reviewer: Vlad Paserin, Research associate, Faculty of Mechanical and Mechatronics Engineering, University of Waterloo, Canada.

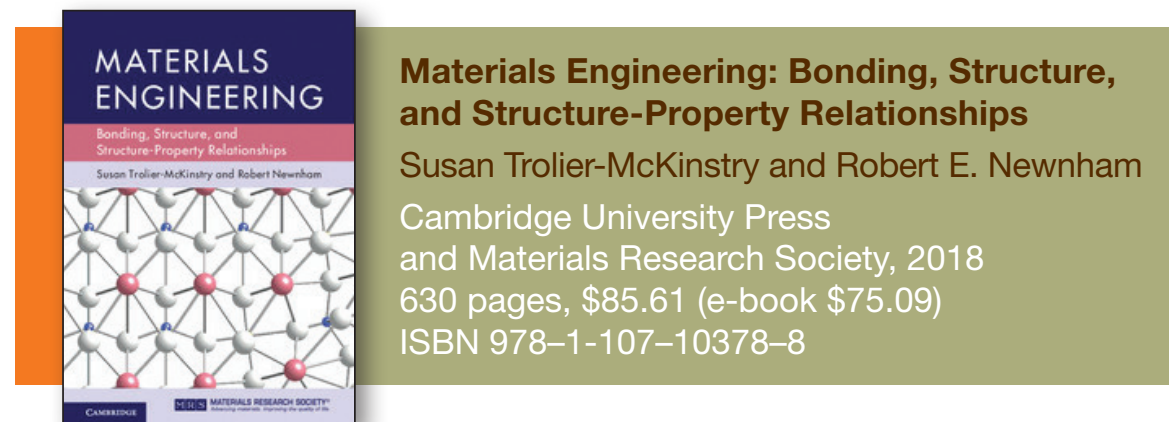

$\mathrm{T}$ The book Materials Engineering has a crystal structure approach to bonding, structure, and how they relate to the properties of materials. However, it does not describe materials processing and microstructure or materials characterization. The subject is covered in 30 chapters (630 pages). The authors use common raw and industrial materials to explain the symmetry relationships in crystals and molecules.

After the introductory chapter, the next three chapters describe the primary raw materials on earth: minerals, water, atmospheric air, and fossil fuels. The bonding between different atoms and ions is discussed in the third chapter and is immediately related to hardness, melting points, and boiling point topics in the fourth chapter. Chapters 5 and 6 discuss the geometry and morphology of crystals, crystal systems, and the theoretical density of crystalline solids. The connecting line of thought is always the crystal structure, or its absence in amorphous materials.

There are several figures in these introductory chapters describing different crystal structures and molecules and pointing to the importance of symmetry before its formal definition in Chapter 7.
The text is fluent and resembles a teacher in a classroom who brings examples related to raw and synthetic materials to pinpoint the theory. Many tables and figures are used to provide hints for the behavior of materials. Whenever possible, the authors include "rules of thumb" related to materials properties.

Chapters 8-12 present covalent, ionic, metallic, molecular, and polymeric materials before going back to the fundamental theory, Pauling's rules, bond valence, structure-field maps, and crystal field theory in Chapters 13 and 14 . Since the text is an introduction to materials engineering, advanced details are not included, but the related physics and chemistry are substituted by figures and tables that provide excellent insight.

Chapters 15 and 16 introduce solid solutions and defects in crystals, with a brief presentation of the Kröger-Vink notation for ionic point defects. The authors finish the general introduction to materials by discussing amorphous 
materials, gases, liquids, and glasses, pointing out the absence of long-range symmetry.

From Chapter 19 onward, the book takes an eclectic approach, mixing chapters on some particular materials, silica and silicates (Chapter 19), and cement and concrete (Chapter 21), with chapters on fundamental properties and different materials as examples, such as an introduction to phase transformations (Chapter 20), surfaces and catalysis (Chapter 22), Neumann's Law and tensor properties (Chapter 23), thermal properties (Chapter 24), diffusion and ionic conductivity (Chapter 25), electrical conductivity (Chapter 26), optical properties (Chapter 27), dielectrics and ferroelectrics (Chapter 28), magnetism (Chapter 29), and mechanical properties (Chapter 30). For all of these chapters, the reasoning line is the symmetry and crystal structure properties, which are the basis for the physics and chemistry of the materials.

The authors rely heavily on figures (488) and tables (83) to teach how materials properties correlate with crystal structures. A table for the Shannon-Prewitt ionic radii is included as an appendix. Unfortunately, the book does not provide references to deepen the knowledge on the presented topics. Exercises are included in most chapters. Solutions are available online for teachers. Readers will profit from a basic knowledge of matrices and simple three-dimensional geometry. A computer program to draw structures will help to learn and visualize the many examples. This book is for undergraduate students or first-year graduate students, but anyone who researches and works with materials will profit from reading this excellent textbook.

Reviewer: Roberto Ribeiro de Avillez, Pontificia Universidade Católica do Rio de Janeiro, Brazil.

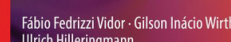
Ulinh Hilleringme an

Zn0 Thin-Film

Transistors for

Cost-Efficient

Flexible

Electronics

\section{ZnO Thin-Film Transistors for Cost-Efficient} Flexible Electronics

Fábio Fedrizzi Vidor, Gilson Inácio Wirth, and Ulrich Hilleringmann

Springer International Publishing AG, 2018

179 pages, $\$ 179.00$ (e-book \$139.00)

ISBN 978-3-319-72555-0

\section{Q}

Clexible electronics is a technology

for assembling electronic circuits by mounting electronic devices on flexible substrates. Flexible electronic assemblies may be manufactured using identical components for rigid printed circuit boards, allowing the board to conform to a desired shape or to flex during its use. $\mathrm{ZnO}$ is a typical Group II-VI semiconductor, with a wide bandgap and high transparency in the visible region. $\mathrm{ZnO}$ is very promising as an active channel layer for thin-film transistors (TFTs). In particular, ZnO TFTs are ideal candidates for producing transparent and flexible electronic products.

This book provides an introduction to $\mathrm{ZnO}$ TFTs for flexible electronics. It comprises seven chapters. Chapter 1 gives a brief introduction to flexible electronics and $\mathrm{ZnO}$ TFTs, as well as the flow of the book. Chapter 2 illustrates the fundamentals of flexible electronics, with an emphasis on ZnO TFTs. Chapter 3 is devoted to the fabrication processes of transistors on solid and flexible substrates, with a general overview of the most used techniques and materials. Chapter 4 discusses the electronic characterization of flexible ZnO TFTs, where along with the current-voltage curves, the TFT behaviors are analyzed in detail. Chapter 5 addresses the performances of circuits integrated with ZnO-based TFTs, including ZnO TFTs and InGaZnO TFTs. Chapter 6 includes improvements for integrated circuits and devices, including the implementation of complementary devices with $n$ - and p-type TFTs. Chapter 7 presents future perspectives for flexible electronics based on ZnO TFTs.
This book provides a general understanding of flexible electronics and progress of $\mathrm{ZnO}$-based TFTs in the field. For flexible electronics, $\mathrm{ZnO}$-based amorphous semiconductors, such as $\mathrm{InGaZnO}$ and $\mathrm{ZnAlSnO}$, have attracted more attention in recent years, which may have great potential for practical applications in transparent and flexible electronics. Transparent, flexible electronics can be considered the next generation of microelectronics, which is still a growing field and in its early stage of development. In this regard, the book gives us an excellent introduction of flexible electronics based on ZnO TFTs. However, the book could improve if more recent achievements on amorphous oxide semiconductors were included.

The authors have compiled information on a set of $\mathrm{ZnO}$-based TFTs for flexible electronics. The contents are well organized and presented. This book is recommended to postgraduate students, researchers, and technologists, especially those who are interested in microelectronics, photonics, and optoelectronics, as well as printed electronics, transparent electronics, and flexible electronics.

Reviewer: Jianguo $\mathbf{L u}$ is an associate professor at Zhejiang University, China.

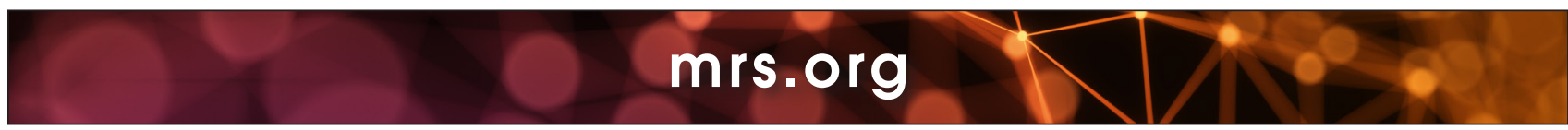

